\title{
Memory Cooperation Optimization Strategies for Multimedia College English Teaching Methodology
}

\author{
Zhang Fenxiang ${ }^{1, a}$ \\ ${ }^{1}$ Hunan Vocational College of Railway Technology, Zhuzhou 412000, China \\ a, zhangfenxiang2013@163.com
}

\begin{abstract}
Keywords: Spontaneous Regulation, multimedia English teaching, Memory Cooperation Optimization
\end{abstract}

\begin{abstract}
This paper introduced the spontaneous regulation and global regulation based more collaboration virtual machine memory management structure for the multimedia teaching in college English teaching, analyzed the most important assurance for dynamic deployment and security isolation for the infrastructure resources. The paper firstly introduced the memory resources abundant state and memory resources state of deflation and then proposed the memory cooperation optimization strategy framework, the structure was proved as high service capacity, high scalability, and lower performance penalty.
\end{abstract}

\section{Introduction}

Though very young, multimedia computing is a kind of application is widely used technology, and in the IT world, all kinds of cloud, arise, some analysts believe that cloud computing represents the enterprise computing mode change. Estimated in the future five years, many giant global manufacturers such as IBM, dell, HP [1], etc. will transfer their product line up to cloud computing. With more and more enterprises will himself to cloud computing, the traditional pursue higher performance CPU chip, the pursuit of more large-scale super computer, hard to improve the performance of a single system of the it industry development pattern will be gradually replaced. Cloud, has become the future development of the IT industry the wind vane [2]. Admittedly, cloud computing is a young technology, many areas have not suitable cloud computing solutions, and many aspects of cloud computing, the key problem has not been solved, cloud computing based on the characteristics of the Internet and make its safety and reliability are a lot of controversy, but these did not influence the cloud computing charm, also have no effect on its future as a computer industry, information industry development direction of the fact, people explore cloud computing enthusiasm is still high. I believe, in the foreseeable future, the IT industry by clouds [1-3] will go.

\section{Virtualization teaching scheme of the specific steps}

In the room of a computer, use the "reduction card" to differentiate afresh partitions, one of the partition need fifty Gb, file system is NTFS [4-5], and divided into a $5 \sim 10 \mathrm{~Gb}$ left and right sides "common material plate", and the rest of the other hard disk partitions, can according to your room set up the planning. In the $50 \mathrm{~GB}$ of partition, the "installation" into the partitions, installs Windows XP with SP2 operating system [4-5], installed operating system after, install driver, reduction card driver and VMware Workstation 7.1 main program. In the install VMware Workstation 7.1 then, create and realize the previous section mentioned each virtual machine, the main steps include create and configuration "basic" virtual machine, create and configuration "cloning link" virtual machine, use VMware Player start the virtual machine, the following three parts were introduced.

\section{1)Primary virtual machine structure}

The so-called primary virtual machine or basic virtual machine is not involved in any experiment content, runs a single operating system, and have a complete software system independent computer virtualization, the virtual machine can install different operating systems, with different virtual hardware facilities for different experimental use, in cloud computing experiments, the foundation of 
these different virtual machine will simulate cloud computing platform in different network running different operating system and performance of different node computer.

\section{2)The copy and reuse of the multimedia teaching node}

Virtual machine cloning is cloud computing environment is an important mechanism, the method of virtual disk and memory Settings snapshot, a virtual machine for rapid replication. Below are some virtual machine cloning method steps:

(1) From Red Hat Enterprise five cloned two virtual machine, it is respectively RHES For TEXT and RHES For X Window, and then start respectively, modify RHES For TEXT virtual machine startup mode as the default enter TEXT [5] interface, and the latter the default boot to the graphics interface, you can modify/etc/inittab [3] configuration files of the id: 5: initdefault operation level to achieve this goal, when id number behind For 3 when the default into the "TEXT" interface, figure number For 5 default into "graphics" interface.

(2) From Windows XP virtual machine, create two clone link of the virtual machine, install the corresponding software. For instance, in PS virtual machine, the installation Dreamweaver, Photoshop software such as shown, as shown in figure 3. After the installation is complete, close the virtual machine [2].

(3) From Windows Server 2003 virtual machine, create four cloning link of virtual machine, including "Oracle" virtual machine, install Oracle database, IIS; SQL virtual machine, install IIS, SQL Server 2000 software; SQL 2005 virtual machine, install SQL Server 2005 software. And, when the virtual machines installed software, and edit a text file or web page, write clear current virtual machine installation system, database name, connection address, the administrator account and password, etc., and modify "desktop" to display the help information.

\section{Introducing of the cloud computing in Memory Cooperation Optimization Strategy}

\section{1)The usage of Memory Cooperation Optimization Strategy}

Cloud computing process has strong vitality and represent the future direction of the development of IT industry, the goal of the people represented in the IT industry, but IT is also need to spread cloud computing in the teaching not only need to implement a solid theory foundation of students, but also requires a combination of practice and more tests, and deep understanding of cloud computing.

\section{2)The problem in traditional English multimedia network teaching platform}

Many computer institutions or Computer College, mechanical room construction is the whole school most of the laboratory construction. The main types of the computer, you need a large number of professional software support learning needs, even if the non-professional students need a lot of software to realize professional experiment. Corresponding to this, a lot of software can only adapt to a specific operating system, construction machinery room, which caused problem: the machinery room, so much manpower and hardware resources of the operating system of the computer frequent change is a big challenge, frequent change the operating system itself is also a kind of hardware damage. On the other hand, this paper discusses cloud computing the teaching purpose, this problem seems to be more serious reality, cloud computing is the Internet - as the foundation, the use of computers in different segment, different physical location, the large scale - a small computer room, apparently impossible, in addition, cloud computing, usually contains the strong performance of high performance computer, these machines, high cost and difficult to maintain, but only partial, such as learning cloud in experiments, will be used to compute the student.

\section{The basic algorithm for Memory Cooperation Optimization Strategy}

\section{1)PM - LB virtual machine deployment algorithm}

In the research deployment algorithm should give full consideration to cloud computing multi-user services environment, according to the virtual machine carries business for different resource dependence degree different (as in scientific computing CPU consumption, used for web server network bandwidth consumption), virtual machine mainly depends on performance become 
user preferences performance, in the allocation of resources, give full of resources reserve space, while the user get a better user experience.

\section{2)Description of the resource performance vector}

For virtual machine deployment first to virtual machine performance for effective monitoring, virtual machine hardware resources mainly include general CPU performance, memory utilization, network connection and configuration state, on host virtual machine basic operation state, etc. In order to standardization characteristics, this paper reference Virtual Machine Manager 2008 technical report for physical server performance evaluation standard, CPU, memory, substitution, hard drives the four fundamental for example, every ten min extraction its condition of average, press the resources characteristics calculation method processing:

$$
\begin{aligned}
& C P U \text { characteristic }=1-\frac{C P U \text { Has usaged }}{(C P U \text { total }-C P U \text { preserved })} \\
& \text { Memory characteristic }=1-\frac{\text { Memory Has usaged }}{(\text { Memorytotal }- \text { Memory preserved })}
\end{aligned}
$$

$$
\begin{aligned}
& \text { Bandwidth characteristic }=1-\frac{\text { bandwidth Has usaged }}{(\text { bandwidthtotal }- \text { bandwidth preserved })} \\
& \text { Hard_Disk characteristic }=1-\frac{\text { Hard_Disk Has usaged }}{(\text { Hard_Disktotal }- \text { Hard_Disk preserved })}
\end{aligned}
$$

Server in the pool all the server through the performance monitoring results of standardized treatment, can establish its performance vector (the 25th, q2,..., qi), including: qi said serve]: first I a performance characteristics, I said used to describe the virtual machine hardware overall performance index number. Will server pool all server UUID composition vector $U=(u 1, u 2$, UN) $t$, including $n$ said the number of physical servers. The server pool UUID and corresponding performance vector can build into a similar Key/Value model performance matrix, such as formula (1) shows:

$$
Q=\left[\begin{array}{ll}
U & V
\end{array}\right]=\left[\begin{array}{ccccc}
u_{1} & q_{11} & q_{12} & \ldots & q_{1 l} \\
\cdot & \cdot & \cdot & & \cdot \\
\cdot & \cdot & \cdot & & \cdot \\
\cdot & \cdot & \cdot & & \cdot \\
u_{n} & q_{n 1} & q_{n 2} & \ldots & q_{n l}
\end{array}\right]
$$

Among them: the matrix each line represents a physical server performance of vector, qij said server UI first $\mathrm{j}$ a performance characteristics.

At the same time according to user submitted for resource scheduling manager virtual machine performance requirement, for each server for standardization, get the virtual machine for each server performance expectations.

Waiting for the deployment of the virtual machine for each physical server expectations can constitute a expect matrix:

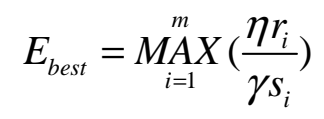

\section{3)The realization of PM - LB algorithm}

PM - LB server found algorithm first calculated for a single virtual machine and physical server performance of the optimum matching, and then calculate the system load balance and comprehensive analysis of the two kinds of calculation methods have a final server selection results. Algorithm and its main body can be divided into the following as below:

\section{a)The match vector calculation}

The difference between the deployment to the performance of each physical server expectations and virtual machine performance characteristics, known as the server to deploy the virtual machine performance as $\Delta q_{i j}=q_{i j}-e_{i j}$. 
Through the expectations matrix resource management server application request submitted to the user's virtual machine, retrieval and server performance monitoring performance indicators, and how to server pool matching amount of matrix can get that:

$$
\Delta Q=\left[\begin{array}{lll}
U & Q-E_{1}
\end{array}\right]=\left[\begin{array}{ll}
U & \Delta Q_{1}
\end{array}\right]=\left[\begin{array}{ccccc}
u_{1} & \Delta q_{11} & \Delta q_{12} & \ldots & \Delta q_{1 l} \\
\cdot & \cdot & \cdot & & \cdot \\
\cdot & \cdot & \cdot & & \cdot \\
\cdot & \cdot & \cdot & & \cdot \\
u_{n} & \Delta q_{n 1} & \Delta q_{n 2} & \ldots & \Delta q_{n l}
\end{array}\right]
$$

When get matrix, each line is negative. However, the performance can't satisfy the demand of a virtual machine; it will be considered as endless node removal from the substrate. Finally, the rest of the m server available server matching quantity matrix:

$$
\Delta Q^{\prime}=\left[U^{\prime} \quad \Delta Q_{1}^{\prime}\right]=\left[\begin{array}{ccccc}
u_{1} & \Delta q_{11} & \Delta q_{12} & \ldots & \Delta q_{1 l} \\
\cdot & \cdot & \cdot & & \cdot \\
\cdot & \cdot & \cdot & & \cdot \\
\cdot & \cdot & \cdot & & \cdot \\
u_{j} & \Delta q_{m 1} & \Delta q_{m 2} & \ldots & \Delta q_{m l}
\end{array}\right]
$$

In order to better meet the needs of the user application, and then matching matrix set weight vector multiplication, which forms a match to determine the carrier as:

$S=\Delta Q_{1}^{\prime} *\left(w_{1}, w_{2}, \ldots, w_{l}\right)^{T}=\left(s_{1}, s_{2}, \ldots, s_{m}\right)^{T}$

With the weight vector, the performance characteristics of the user's preference is given less weight value, this is in the distance calculation order to reduce the limit, also can make server greater efficiency as the preference its features are reserved space.

\section{The analysis about the performance}

By using CloudSim, we extended the compiler simulation program and the simulation is completed, we will be able to Verity by two parameters, the performance of the algorithm can be used to compare the experiment.

A. During the operation of the virtual machine server (as server opens) N. Value, the smaller the number of servers, turns to the less, save more energy.

B. Load balancing factor, the variance of the load by seeking to determine the vector to determine the load balance.

Thus, the simulation results are shown as the below two figures:
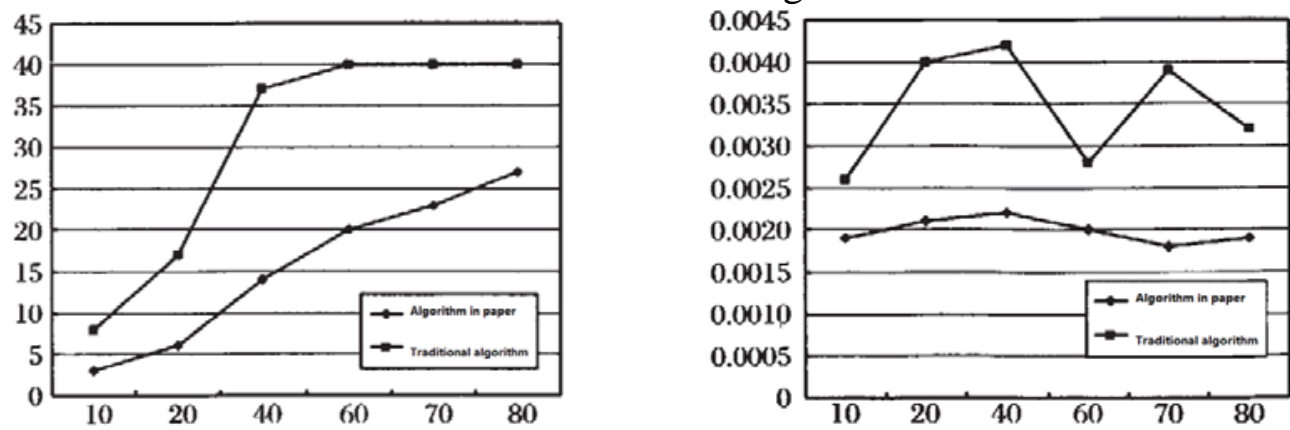

Figure 1. The number of open server comparision Figure 2. Open server load variance Comparison

From the experimental results we can confirm that: PM - LB algorithm can greatly reduce the system server open shoulder quantity, which reduces the cost of the system resources. At the same time, it is able to maintain stability in the low range, in order to achieve good load effect system load. Therefore, we can draw the conclusion that this algorithm can meet the cloud computing environment of resource usage and system load demand and virtual machine deployment. 


\section{Conclusion}

During the process of English teaching, multimedia assisted teaching has its gained popularity, but the limitations of the traditional algorithm still exist. To improve teaching method, this paper introduces a virtual machine in the Memory Cooperation Optimization Strategies. In this paper, the initial deployment of virtual machine program and the algorithm was also put forward. This paper first introduces the abstract to physical server and virtual machine performance vector, then on the basis of the performance of the carrier, the carrier and the judges were matched load balancing judgment vector calculation performance. As the result of comprehensive management for the ultimate deployment, consisting of two vectors, the experiments and analysis show that the algorithm can solve the problem with proper load balance as well as saving resources.

\section{Acknowledgements}

The twelfth five-year planning topics of Hunan education sciences in 2013.

\section{References}

[1] Hiromichi Kobashi, Shigeo Kawata, Yasuhiko Manabe, Masami Matsumoto, Hitohide Usami, "PSE (Problem Solving Environment) Park Engines toward a Meta-PSE on Cloud in Scientific Computing", JCIT, Vol. 6, No. 11, pp. $141 \sim$ 154, 2011.

[2] Mohamad Mohamad, Mohd Helmy i, Cik Fazilah Hibadullah, "An Investigation into Influence Factor of Student Teaching condition improvement Using Association Rule Mining", New Generation Teaching, Vol. 5, No. 11, pp. 99 107, 2009.

[3] Yazed Al-Sawi, Ajlan Al-Ajlan, Khalid Al-Drewiesh and Abdullah Bajahzer, "An Efficient Search Agent Software for Multi-Agent Systems", JDCTA, Vol. 3, No. 1, pp. 73 87, 2009.

[4] Mohammed Abdulraoof Abdulrazzaq Al-Sabbagh, "Enhancing The Programming of VMware ", Virtual World, Vol. 1, No. 1, pp. 2 10, 2006.

[5] Peng Lu, Dongdai Zhou, Qilan Chen, Shaochun Zhong, "Design of the Public Service Platform in Digital Campus based on "Cloud + Agent" Mode", JDCTA, Vol. 6, No. 18, pp. 418 426, 2012. 